\title{
Real-time color-frame animation for visual psychophysics on the Macintosh computer
}

\author{
SCOTT B. STEINMAN \\ University of Missouri, St. Louis, Missouri \\ and \\ MARK NAWROT \\ Vanderbilt University, Nashville, Tennessee
}

\begin{abstract}
Psychophysical experiments involving moving stimuli require the rapid presentation of animated sequences of images. Although the Macintosh computer is widely used as a color graphics computer in research laboratories, its animation capabilities are generally ignored because of the speed limitations of drawing to the screen. New off-screen color graphics structures help to avoid the speed limitations so that real-time color or gray-scale visual motion stimuli may be generated. Precomputed animation frames are stored in off-screen memory and then rapidly transferred to the screen sequentially. The off-screen graphics structures may also be saved to disk in "Picture" form as "resources" for later retrieval and playback, allowing the experimenter to build in advance a collection of moving stimuli to use in future experiments. Code examples in the $\mathrm{C}$ programming language are provided, and the relative strengths and weaknesses of Macintosh color-frame animation for psychophysical experimentation are discussed.
\end{abstract}

The Macintosh II series extends the graphics capabilities of the Macintosh computer into the color domain. This has led to the adoption of the Macintosh in many vision laboratories for color graphics applications such as data presentation and image processing. However, few laboratories use the Macintosh for conducting visual psychophysical experiments.

Psychophysical experiments involving moving or changing stimuli require the presentation of animated sequences of images. It has generally been thought that the Macintosh computer could not be used for real-time color (or gray-scale) animation. Unlike computers such as the Commodore Amiga, the Macintosh lacks dedicated graphics coprocessor chips that speed up critical drawing operations. On Amiga and IBM-compatible computers, an animation sequence's images may be created and displayed in real time on a frame-by-frame basis by using video memory page-flipping techniques (Anstis \& Paradiso, 1989; Wenderoth, 1990). The lack of a coprocessor or dual video memory banks in the Macintosh places the burden of generating images directly on the central processing unit (CPU); it must execute QuickDraw graphics routines on the fly. Macintosh QuickDraw routines are far from what their name implies; they may be quite slow. Although recent advances such as the Apple 8-24GC graphics accelerator board do speed up some drawing routines, QuickDraw operations cannot be ex-

The authors would like to thank Dr. John Baro for many helpful discussions on the Macintosh Toolbox and for comments on early drafts of this manuscript. Correspondence should be addressed to Scott B. Steinman, School of Optometry, University of Missouri, 8001 Natural Bridge Rd., St. Louis, MO 63121. ecuted quickly enough for both real-time generation and display of animation frames.

The time overhead of executing QuickDraw drawing routines may be circumvented by generating the individual frames of the animation sequence in advance. These precomputed images are stored in off-screen pixel mapsgraphics representations that reside in memory. When the animation sequence must be shown to the observer, each frame is quickly displayed by transferring the frame's pixel map contents to the active graphics device's memory with the ToolBox CopyBits routine. By reducing the graphics operations required during each animation frame interval to a single copying procedure, the animation may be carried out in real time.

This article describes several interrelated animation techniques written for the Macintosh computer for the generation of an animation sequence, the saving of animation frames to disk for later retrieval, and the displaying of the animation sequence in real-time by using Toolbox Vertical Retrace Manager timing routines (Rensick, 1990). The frame images are written to disk files as a collection of color picture resources, which may be created with commercial paint programs and the ResEdit resource editor (Apple Computer, Inc.) Other resources stored in the disk files preserve the color environment and pertinent experimental parameters for playback of the animation. Code fragments in the $\mathrm{C}$ programming language ${ }^{1}$ are provided, and the relative strengths and weaknesses of Macintosh color animation for psychophysical experimentation are discussed.

It should be stressed that very little information about animation on the Macintosh computer is available. The code described here integrates the function of several 
Toolbox Managers specifically to facilitate and optimize the presentation and storage of animated color images. More importantly, many of the techniques discussed in this article make use of features of the Macintosh Toolbox and the 32-bit QuickDraw that are not documented in Apple Computer's Inside Macintosh $(1985,1991)$ manuals or the Macintosh Technical Notes (1984-1991).

\section{Background}

Until recently, programmers have had to make significant modifications to black-and-white graphics programs to take advantage of the color environment found on the Macintosh II computer series. Programs written for older Macintosh models could use direct BitMap representations of the arrays of pixels to be shown on the monitor screen. With the introduction of color to the Macintosh, programmers now had to keep track of complicated interrelationships between color pixel maps, color tables, inverse color tables, graphics device structures, and color graphics ports. Color graphics programming proved to be so difficult that few programmers abandoned black-and-white graphics.

Apple Computer has now provided simpler ways of accessing the new color environment. One of these is the GWorld off-screen graphics environment that is part of 32-bit QuickDraw. The other is the Palette Manager, which simplifies the specification of color. Before delving into the workings of the real-time animation code, a brief introduction to some aspects of GWorlds and the Palette Manager is required. The Resource Manager, which is used to store the animation sequence in a disk file, is also discussed. The reader is referred to Rensick (1990) for a discussion of the Vertical Retrace Manager, used here to accurately time the playback of the animation sequence.

\section{The GWorld Off-Screen Graphics Environment}

Real-time animation has always been difficult on the Macintosh, even when black-and-white (1-bit) graphics were employed. To display an animated image, a program had to attempt to draw each animation frame within a screen refresh interval (15 msec on Macintosh II machines). If the drawing time exceeded this interval, the image would appear to flash or shear or, even worse, the animation would slow down to a crawl. QuickDraw drawing routines are relatively slow, preventing direct drawing of an animation frame's contents in real time, especially if the image is complex. To represent color, these drawing routines must cope with several bitplanes, further slowing the drawing process. Programmers found that they could bypass these speed constraints with off-screen BitMaps (black-and-white) or PixMaps (color), undisplayed representations of a screen image that reside purely in memory. Off-screen graphics are most often used to carry out single rapid alterations to the screen image. Drawing to off-screen memory is faster than drawing to the screen, and the contents of this memory may then be copied quickly to the screen. For example, a graphics file may be read from disk and drawn into off-screen memory, then copied to the screen by using the ToolBox CopyBits routine. Similarly, animation can be accelerated by drawing each frame's image in advance to an element of an array of off-screen BitMaps (or PixMaps), then quickly copying the contents of each BitMap to the screen in turn. Each call to CopyBits could be executed within one screen refresh interval.

The GWorld graphics world construct provided by 32bit QuickDraw is an enhanced equivalent of the BitMap or PixMap record. It not only contains a map of an image's pixels and their colors but also includes its own color table (CTable) structure and graphics device (GDevice) structure. The GWorld encapsulates and insulates the inner workings of these structures from the user. Instead of having to create and manipulate all of these individual color structures, the programmer simply creates a GWorld graphics environment with a call to the NewGWorld routine and destroys it with a call to DisposeGWorld. In the case of color-frame animation, an array of GWorlds may be assigned to hold the images for each frame of an animation sequence. Rather than attempting to draw each animation frame directly on the screen, we first draw them instead into an array of off-screen GWorld structures. When the animation sequence is to be displayed, all we need do is copy their contents to the screen in real time.

The GWorld structure does more than just store the animation frame images. It also serves to further accelerate the animation display. When off-screen images are copied from memory to the screen, the speed of the transfer is affected not only by image size and number of colors but also by the position of the image. Images whose PixMap's leftmost pixels are aligned to long-word boundaries in memory are transferred by CopyBits faster than those that are not. In addition, if the image PixMap's leftmost pixel falls on a different byte or bit in memory than does its desired screen location, CopyBits works even slower. The GWorld structure allows some control over the speed of image transfers by correcting for these factors. If the programmer converts the GWorld bounding rectangle to global QuickDraw coordinates before passing it to NewGWorld (see Apple Computer, 1991), the NewGWorld routine will automatically optimize the graphics image position within the GWorld pixel map so that CopyBits will work as quickly as possible. This optimization does not affect where the graphics will appear on the screen but only the internal representation of the graphics image within the GWorld so that the image may be accessed faster.

\section{The Palette Manager}

Display monitors are represented in the Macintosh operating system by a graphics device (GDevice) data structure that contains, among other things, the color environment for that monitor. This ensures that each monitor of a multiple-monitor system preserves its own individual color environment. Each color graphics device structure contains two color look-up tables, which are the 
means by which Color QuickDraw determines the actual color used for drawing. The first is a direct color lookup table ("clut") containing a list of color specifications in RGB format that may be directly displayed on that graphics device. The second is an inverse color look-up table that helps the system cope with other colors. If you request a drawing color that is not in the direct color table, Color QuickDraw will use the inverse color table to determine the best possible match to the color you have requested. If a close match cannot be made, the direct color table may be changed, which sometimes is an undesirable side effect. To explicitly change the color look-up table, the programmer must use a color table structure (CTable, not to be confused with a clut) to provide new color definitions and update both the direct and inverse color look-up tables with Color Manager routines.

Off-screen graphics are also "displayed" by using a graphics device structure, but this device does not really exist in a physical sense. When a GWorld is created with NewGWorld, the programmer may choose to create a new (off-screen) graphics device to hold the GWorld's image or to use a preexisting graphics device. This option allows the creation of one graphics device for an array of GWorlds. The programmer must also provide a color table (CTable) structure for the GWorld's graphics device, either explicitly by passing a color table structure to NewGWorld or implicitly by using the color table of the physical graphics device to which the GWorld's image will ultimately be transferred.

Color Manager calls can be used to update the color look-up table when changes to the color environment are necessary, for example, when the target contrast must be modified. However, working with color tables directly is not only discouraged by Apple Computer but also requires that several data structures such as inverse color tables be simultaneously kept up to date (see Apple Computer, 1991). It is far easier to rapidly update the color environments with the newer Palette Manager routines. Palettes are a higher level form of color information that embody all of the Color Manager data and routines but are easier to use since they automatically handle both the direct and inverse color look-up tables (for a full discussion of palettes, see Baro \& Hughes, 1991). When a palette entry's color is changed, every pixel previously drawn with that palette entry is instantly changed to the new color. Because GWorlds contain graphics device information, the Palette Manager routines are made available to them. In the frame animation code, parallel updates of the animation display window and each animation frame are accomplished easily by using the same Palette Manager routines. This means that the color environment of the display window and the animation frames is always kept synchronized so the background color of each is always the same. The frame's image blends seamlessly into the window background.

\section{Pictures and Resource "Files"}

Macintosh graphics may be specified in two ways. One method, which has been discussed above, is the pixel map
(PixMap), in which the graphics are represented as arrays of pixels on the screen. Although this is the most direct way to represent and store graphics images, files containing color pixel maps require a lot of disk space. A second way of representing graphics is not in terms of individual pixels, but as a set of drawing instructions that are used to draw the graphics. The Macintosh ToolBox contains routines that allow the programmer to store a list of drawing instructions in a Picture record, which may be used at any time to reconstruct the graphics image.

Single color graphics images are typically stored as Pictures in data files with the PICT file format (color graphics actually use the newer PICT2 format). The equivalent file format for animation sequences is the PICS file, which is essentially a collection of PICT images with added information such as playback speed. These standard file formats pose some problems for the storage of visual psychophysical stimuli, which are typically presented on carefully controlled backgrounds.

Part of the problem is due to the structure of a PICT or PICS file. Macintosh files contain two parts or "forks," each of which the programmer may choose to use or not use. The data fork contains raw data that are essentially unstructured streams of data bytes. It is the program that creates any structure in this data. The resource fork uses collections of data called resources that are stored in specific formats, some of which have been formalized by Apple Computer. Resources may be modified independently of program code by using the ResEdit resource editor (Apple Computer, Cupertino, CA). ResEdit allows us to view and change the data in some resources in graphical form exactly as they would be presented on the screen, giving the programmer more flexibility in understanding and using the data.

Both the PICT and PICS graphics files use only the data fork of a disk file. Resources at one time were limited in size to 32-K, which prevented the storage of large pictures or color pictures to disk as resources. Although this limitation no longer applies, the storage format for pictures and animation sequences has not changed. The resource forks of PICT and PICS files are therefore empty-no separate color table (clut) or palette (pltt) resources are retained. Palette information is encoded directly into each PICT's data. If a programmer wishes to extract the color information in the PICT in order to set the palette of the window that will display the picture, the image must be picked apart piecemeal with a complicated and cryptic custom QuickDraw routine written by the programmer (Sheets, 1988). Similarly, special techniques must be used just to read and write the picture data in PICT files (see Apple Computer, 1991).

A more straightforward means of saving and restoring the individual graphics images and the color environment is to save the images as standard PICT-type resources, and to store along with them a standard color table (clut) resource. This may reduce direct compatibility with commercial paint packages that use data-fork-based PICT files, but the programmer may more easily store custom graphics created by a psychophysics program as resources 
for later use. In addition, the ResEdit resource editor may be used in conjunction with commercial paint programs to transfer graphics created by the paint program to PICT resources via the clipboard. Generating stimulus images with a paint program enables the rapid preparation of a battery of test stimuli for a wide range of psychophysical experiments (Anstis \& Paradiso, 1989).

Resources may also be used to store the color information present in the color table of the off-screen GWorlds. This allows the psychophysics program that will display the animated visual stimulus to set the screen background color to that of the animation frame. This is very important in psychophysical experiments in which a stimulus must be shown on a fixed luminance background, for example, sinusoidal grating patches on a gray background. By storing and retrieving the color table with resources, the proper background luminance is maintained every time the stimulus is presented.

Resources also provide an elegant way to store relevant experimental parameters in the animation file. A custom experimental parameter resource may be created to store a $\mathrm{C}$ language structure (or Pascal record) containing the desired experimental settings such as animation frame presentation rate, stimulus size and color, background color, psychophysical method to be used, and so forth. This resource is read from the file before the animation is to be presented to the subject, which serves to ensure the proper settings for conducting the experiment. An added benefit of storing the experimental parameters as resources is that this data may be easily examined and modified later in the animation file so that new permutations of stimuli may be created. ResEdit allows the creation of custom resource templates so that the programmer may determine exactly how he or she wants to view the data. The experimental parameter data may be set with a template to be displayed as a series of text entry fields, in which a meaningful label of the data is placed next to the field. For example, the frameDelay parameter's value would be displayed in its own field with the label "Frame Delay" next to it. The data may be directly modified by using the text entry fields. Were the experimental parameters stored in the data fork of the animation file, modifications would have to be made with a hexadecimal file editor with full knowledge of which data file byte contained each experimental parameter.

\section{The Color Animation Code}

Using the techniques outlined above, 100 frames of animation containing a $200 \times 200$ pixel frame area in 4-bit (16-color) gray scale have been animated in real time ${ }^{2}$ on a Mac IIfx computer. As discussed below in the Conclusions section, it is possible to animate even larger images in real time. The code fragments to be examined are part of a larger visual psychophysics experiment program that is composed of two interdependent parts. The first part calculates and draws the animation frames, which are then stored to a disk file. The second part presents the moving stimuli and measures motion direction or velocity discrim- ination thresholds. The full program implements many features that cannot be discussed within the space constraints of this article. Among them is a batch-processing mechanism with which a series of experiments, each composed of several presentations of a moving stimulus, may be conducted. The observer's responses are recorded with the keyboard. The full program therefore allows the experimenter to collect a sizable block of psychophysical data automatically.

It should also be noted that the code fragments listed here do not contain the event-handling routines or errortrapping code (including handle and pointer initialization before memory allocation) required by Macintosh application programs. In addition, several crucial variables are shown here as global variables rather than as function input and output parameters in order to simplify the function declarations. The use of global variables should be limited in actual programs. To conserve space, the external variable declarations of each listing are omitted. The reader is directed to Listing 1 for the initial declarations of these variables.

Listing 1 is a segment of the file that typically would contain the code's main function. This listing opens with a list of variable declarations that deal with the construction of an animation sequence, some of which will be described here. The most important variable is an array of pointers to GWorlds called frames, which holds the animation sequence frame images in pixel map form. These pixel maps are displayed on the screen when the animation is to be shown to the subject during the course of an experiment. The following declaration (animSequence) is a handle to a PICS-format animation structure defined in Listing 2 (Sheets, 1991). The structure contains an array of animation frames in Picture format for storage in an animation disk file. Next are grayPalette, a handle to a palette, and grayCTable, a handle to a color table, both of which will specify the colors used in the display window and in animation frames. Two Rect declarations hold drawing areas for the animation frame images. DrawArea is the extent of a frame image in the display window in which the animated stimulus will be presented. Bounds defines the extent of the frame image stored within the pixel map of each GWorld in local QuickDraw coordinates. The complete animation program was written to use complex gray-scale stimuli whose contrast levels could be set individually by the experimenter. Two variables, targetGray and bkgndGray, are declared here for illustration to specify the target and background gray values. Since gray-scale colors are defined with equal red, green, and blue components, a single number may be used to represent all three components. The gray levels are permitted values from 0-255 because Apple graphics boards can display 256 possible gray levels. The gray value is simply multiplied by 256 to yield the RGB setting of 0-65535 required by Color QuickDraw. FrameDelay is the number of vertical blank intervals for which each animation frame is displayed. The variable currColordepth is the monitor color depth at the onset of the program. 
The color depth will be changed by the animation code and must be restored to its initial settings at the completion of the code. Finally, we include a flag to indicate whether the code is running on a Mac IIci, since an additional instruction is required for this machine in Listing 5 .

The CheckEnvironment function uses the System 7.0 Gestalt Manager to determine the characteristics of the machine that is running the animation code. Since the Slot Manager is required for the proper execution of the animation display interrupt code (Listing 5), machines that lack NuBus slots are detected and prevented from running the remaining code. If the machine is a Mac IIci, the Macllci flag is set to true. Similarly, routines included in 32-bit QuickDraw are used in the animation code, so machines without access to 32-bit QuickDraw cannot run the code. Finally, the SetMonitorDepth function is called to set the proper color environment for the animation code.

The InitParameters function initializes major experimental parameters and allocates memory for storing the animation sequence (in Picture form) in the animSequence structure. Since animation requires large amounts of memory, the code depends heavily upon memory allocation to allow the Memory Manager to make the most efficient use of available memory. The function CleanUp frees the memory allocated for the animation sequences in pixel map and Picture formats, the color table, and the palette. It also restores the monitor color depth setting that was present at the onset of the animation code. This function is normally called on program exit, either when the experimenter has completed constructing stimuli or running an experiment, or if a serious error such as insufficient memory occurs during run time.

The code header file, shown in Listing 2, begins with a listing of resource ID number constants for the frame images, color environment, and parameters that will be saved in a disk file in Listing 6 . The following resource ID number, Gray4BitCTableID, identifies one color table resource stored in the Macintosh ROM. The ROM contains several color table resources containing grayscale "ramps" whose ID number is simply the color depth plus 32. In the present code, a depth of 4 bits (16 gray levels) is used, so the ROM gray-scale resource ID number is 4 plus 32, or 36 . Following this is a series of colorspecific definitions, including palette index numbers for the colors to be defined in the code. The first entry of a palette (entry 0) is defined by Apple Computer to be white, whereas the last entry (in the 16 color palette, entry number 15 ) must be black. The remaining palette entries (1-14) may be used by the animation code, although only two entries (PalBkgnd and PalTarget, used for the background and target colors, respectively) are used in the present code fragments. Finally, animation-specific constants are defined. MaxFrames is the maximum allowable number of animation frames. Although this has been defined here as 100 frames, the animation sequence may be composed of any number of frames, limited only by the amount of available memory and disk space. The speed of the animation is limited only by the color depth and image size, not by the number of animation frames.
The header file also contains some important type definitions that define the structures used to store the Pictureformat animation frames and the experimental parameters. These structures will be saved in their entirety as resources in the animation file. The first two type definitions are structures used for saving PICS-format animation files. The reader is directed to the paper by Sheets (1991) for further information on the PICS animation format. Although some of the fields of these structures are not used by the present code, they are included for future compatibility with commercial animation programs. The PICSInfoRec contains animation parameters such as the size of an animation frame, the playback speed, and the color depth. The PICSRec structure, usually stored in the data fork of PICS animation files, contains an array of animation frames called frames in Picture format. Next, the experimental parameter structure, ExpParamRec, is defined to contain duplications of the (global) variables specifying the experimental conditions under which the animation will be displayed. The ExpParamRec structure could have been used in place of the individual global variables throughout the code. However, some variables, such as frameDelay, should be kept in a global variable outside of a structure. The time-critical video interrupt routines (Listing 5) must access variables quickly, so the extra machine instruction needed to access a structure member's memory location is avoided by keeping these variables outside of a structure. A global variable is used for frameDelay because interrupt routines may only pass variables by means of global variables.

The color environment is manipulated via the code in Listing 3. The SetMonitorDepth function sets the proper color depth for running the animation code. In the animation code presented in this paper, we use a color depth of 4 bits ( 16 colors) to allow a larger frame area to be animated. Before changing the depth to 4 bits, we save the initial depth setting so that we may restore it when we have completed running the animation code. InitPalette sets up the gray-scale palette and color table to be shared by the display window (mainWindow) and the animation frame GWorlds ( frames). A gray-scale ramp color table is obtained from the Macintosh system ROM with a call to GetCTable and saved in grayCTable. The NewPalette then creates a gray-scale palette from grayCTable. The pmTolerant and pmExplicit flags tell the Palette Manager how the palette will be used (see van Brink, 1990, and Inside Macintosh, Volume VI, Apple Computer, 1985, for a full discussion of Palette Manager routines). They dictate how a color that is requested from the palette for a drawing operation relates to the colors present in the display device's color look-up table. The pmTolerant flag states that a given palette entry has a "close match" present in the look-up table (precisely how close it must be is given in the last parameter passed to NewPalette). The pmExplicit flag states that a requested palette entry has a corresponding color in the device color look-up table at the same entry number in the table. These two flags (and the last NewPalette parameter) in a sense describe the degree of "noise" that is accepted in the colors the 
system will actually provide when the programmer requests a specific color. Although it is not intuitive, a combination of the pmTolerant and pmExplicit flags is required for proper reconstruction of the color environment from our animation file. The flag combination ensures that a color requested from the palette exactly matches its corresponding device look-up table color entry; in other words, the color asked for is the color obtained. The call to NSetPalette assigns the new grayPalette palette to a specific window-in this case, the mainWindow window in which the animation will be displayed. Finally, the palette is activated for use in the display window.

The UpdateWindowColors function resets the color palette when the experimenter requests a new background or target color. It directly replaces specific color entries in the palette with new color values. SetEntryColor places the new color value (in RGBColor form) at a given entry position (PalTarget or PalBkgnd) in the grayPalette palette. The changes to the palette are then mirrored in the grayCTable color table that is used to create animation frame GWorlds via the Palette2CTab routine. Finally, the updated palette is activated for use. The corresponding changes to the GWorlds' color environment are handled by the UpdateGWorldColors function. This function treats each animation frame GWorld as a color port with its own palette. The two calls surrounding the Palette Manager routine calls simply save the current drawing port and device before using the GWorlds, restoring them afterwards.

Listing 4 is the heart of the animation code. The ReadyFilm function allocates and initializes the off-screen GWorlds that hold the animation frames in pixel map form for later display. First, the rectangles that define the bounds of the image drawing and storage areas are calculated. The extent of the frame image stored in the GWorlds, stored in bounds, is defined in local QuickDraw coordinates. As discussed above in the section about GWorld off-screen graphics environments, several optimizations are performed on the GWorld image if the bounding rectangle is converted to global QuickDraw coordinates before it is passed to NewGWorld. Therefore, the bounding rectangle is converted to global coordinates and stored in the globBounds variable. The display window palette is then updated to ensure that the GWorlds are created with the use of the most recent color values. An array of new GWorlds is created with the use of the NewGWorld routine (after any previously used GWorlds are destroyed). The first GWorld in the array will by default be assigned an off-screen graphics device by passing no flags to NewGWorld. By passing no color table handle to NewGWorld and specifying a color depth of zero, NewGWorld is forced to use the color table and depth of the graphics device on which the animation will be displayed (see Ortiz, 1990, for a description of NewGWorld input parameters). We have already set the color depth to 4 (for 16 colors) with the SetMonitorDepth function. When we did this, the monitor graphics device's color table was set to the default 4-bit color table. It is this color table that NewGWorld uses. The NoPurgePixels routine ensures that the pixel map memory of the GWorld will not be destroyed without our knowledge if the Memory Manager must free up memory during program execution. The remaining GWorlds in the frames array are created by using the GDevice of the first GWorld, obtained with the GetGWorldDevice routine. In this manner, all of the GWorlds share the same graphics device, which saves memory.

The FilmFrames function creates the individual animation frame images. It first calls a CalcOrigin function (not shown in the code listings) that simply calculates the screen position at which to draw the animation frame. In the complete animation program, the position is either the center of the screen if the stimulus is to be viewed with central vision or at a position at a given offset from the screen center if it is to be viewed peripherally. The ReadyFilm function is used to prepare the GWorlds to house the animation frames. Any preexisting Picture-format animation frames are destroyed, since they will be replaced with new versions shortly. The current drawing port and device are saved by a call to GetGWorld. To draw directly into the GWorld pixel maps, the memory handle that points to the pixel map must be locked by using the LockPixels routine, just as other memory handles in Macintosh programs must be locked with the HLock routine. The current drawing port and device are then set to those of the GWorld we wish to draw in with SetGWorld. Next, the DrawFrames function, which does the actual drawing, is called. Finally, the previous drawing port and device are restored, and the GWorld pixel map memory handle is unlocked. The drawing-pen colors are reset to black and white for the proper operation of CopyBits (CopyBits will alter the colors of the image it transfers if the pen colors are not set to these values).

DrawFrames carries out the actual drawing of the animation frame images into both the GWorld pixel maps (for display of the animation sequence) and the Picture structures (to be saved in the animation file on disk). First, the image to be stored in the GWorld is drawn. The drawing-pen size is set, and the GWorld pixel map contents are erased by using the background color. The pen color is then set to the target color, and the target is drawn. To save the animation frame in Picture format in a resource, a Picture of the frame must be created. Unfortunately, Picture instructions do not understand Palette Manager calls, and the older Color Manager equivalents must be used. To do so, the target and background colors must first be converted to the RGBColor form understood by the Color Manager. The GetEntryColor routine converts palette entries into RGBColors. A Picture structure is then opened for filling with drawing instructions by using the OpenPicture call. The Picture will be stored in one frame field of the animSequence structure. All of the frame drawing instructions are then repeated by using the Color Manager routine RGBForeColor instead of Pm- 
ForeColor. The Picture sequence is then closed by means of ClosePicture.

The PlayFrames function is called when the animation is to be presented to the observer during psychophysical threshold measurement. It first hides the mouse pointer from view and initializes the frameIndex frame counter to indicate the first frame of the animation sequence. The InstallVBlankAnim function call initiates the animation display. An empty loop is now entered that waits until the frame counter frameIndex has reached the final frame number, whereupon the entire animation sequence has been shown. The empty loop is exited, and the RemoveVBlankAnim function is called to prevent further display of the animation sequence. The background is then erased by calling the ResetBackground function (not listed here), and the mouse pointer is revealed again.

Listing 5 contains the video interrupt-based animation timing routines. This discussion will concentrate on the code that is specific to presenting color-frame animation. (For a full discussion of millisecond and vertical retrace timing, consult Rensick, 1990.) InstallVBlankAnim sets up the slot interrupt task and the video interrupt task in which we will do the actual animation. The video task procedure address is set to that of the VideoProc procedure, which performs the animation. The vblCount field of the video task structure, which determines how often the VideoProc will be executed, is given a value of frameDelay. This means that once every frameDelay vertical blanks, a new animation frame will be displayed. The value of vblCount therefore partially determines the velocity of the animation. The global index to the current animation frame to be displayed (frameIndex) is initialized to zero. We must also set up a slot interrupt routine for monitors attached to NuBus graphics boards. The slot interrupt task will be called whenever the graphics board generates an interrupt signal on the NuBus-that is, once every vertical retrace. The remaining code in this function automatically determines the slot number of the $\mathrm{Nu}$ Bus slot containing the graphics board. This code works as well on machines with built-in video ports such as the Mac IIsi and Mac IIci. The corresponding function, RemoveVBlankAnim, removes the video interrupt task from the interrupt queue, restoring the interrupt queue to its original state. The VideoProc function is the core of the real-time animation technique. This function is called once every frameDelay vertical blanks. The call to LockPixels that is required for accessing the GWorld's pixel map is made. A single CopyBits call follows in which the contents of the GWorld image is transferred to screen memory. The GWorld pixel map is then unlocked again. This completes the activities required by the current animation frame, so the next frame is set up by incrementing the frameIndex frame counter and resetting the vblCount to wait until another frameDelay screen refreshes before calling VideoProc again. The SlotProc function, called once every slot interrupt, simply calls the video blank interrupt task-that is, VideoProc.
The last code fragment presented here, Listing 6, shows how the newly created animation sequence, color environment, and experimental parameters are stored to disk and retrieved later when the animation sequence is displayed to an observer during an experiment. WriteImage is responsible for storing this information to a disk file. Some of the code within this function is required because of a poorly documented feature of the Resource Manager. When a resource file is opened, a table of the resources in the file, called a "resource map," is created, which effectively links each resource in the file to a handle to a data structure in memory that holds the contents of the resource. For example, if the resource file contains a PICT resource, the resource map will contain a handle to a Picture structure in memory that will hold the contents of the PICT resource. The code listed here creates a resource file and then calls AddResource, which adds handles to an experimental parameter structure, color table, and each animation frame Picture to the resource map of the file. When CloseResFile is later called, this information is automatically stored as resources in the disk file. However, CloseResFile also destroys the resource map, which has the side effect of freeing the memory handles in the resource map. These handles point to animation frame Pictures, a color table and the experimental parameter structure, so these structures are now destroyed. Although this is not a problem for the data structures that are not used later, destruction of the color table has adverse effects on program execution. Therefore, a copy of the color table is created for storage in the resource file. The copy is destroyed when CloseResFile frees the resource map, leaving the original color table intact.

WriteImage first allocates storage for a ExpParamRec structure to hold duplicate values of the experimental parameter variables declared in Listing 1 . These variables must be encased in a structure to be saved in one block to a resource. A resource file is then created and opened for use. The call to SetVol sets the current directory to that chosen with a Macintosh Standard File Package "Save File" dialog box (the code for selecting a file directory and name is not shown here). CreateResFile then creates a resource file with the name chosen in the "Save File" dialog box, and OpenResFile opens this newly created resource file. Each frame of the animation sequence, stored in Picture format in the animSequence structure's frame array field, is transferred to a separate resource by a call to AddResource. AddResource is passed a handle to an animation frame Picture, a definition of the type of resource to be saved (in this case, a PICT Picture resource), a unique ID number for the resource as defined in the header file shown in Listing 2 , and an optional name for the resource that helps the programmer when using ResEdit. Similar calls to AddResource are then made to transfer the experimental parameter structure and a copy of the color table to resources. CloseResFile automatically forces the resources saved to the disk to be updated, releases the file's resource map, and closes 
the resource file. Unfortunately, when the handles in the resource map are freed, the handles are not reset to "null" (the value of an unused handle or pointer). If a memoryfreeing function were called at any time after saving the resource file, and these handles were not set to null, the function would attempt to free the memory locations contained in the handles, which the Memory Manager could by now have assigned to hold other information. The WriteImage function therefore explicitly sets these handles to null to prevent a potential catastrophe.

Reading a previously saved animation sequence file involves most of the steps carried out in WriteImage in reverse order. The ReadImage function starts by freeing memory that has been previously allocated for animation frames, the palette, and the color table so that these may be replaced by those stored in the disk file. The resource file selected by a Standard File Package "Open File" dia$\log$ box is then opened, and a color table and an experimental parameter structure are created and initialized by reading the corresponding resources from the animation file. The color table is obtained by calling GetCTable, which reads a color table resource of the requested ID number from a file and creates a color table structure to house it. DetachResource allows the use of the structure (now pointed to by grayCTable) to remain in memory and not be destroyed after the resource file is closed and the file's resource map is freed. A new palette is created from this color table. Because the experimental parameter structure is stored in a custom resource, there is no dedicated ToolBox routine for reading the contents of this resource. Instead, the generic Get1Resource routine is used to create and initialize the memory that will store the contents of this custom ExPr resource. The memory chunk returned by this routine is typecast to an experimental parameter structure. The array of animation frames (in Picture format) is read from the file by using the GetPicture routine and stored in the frames field of an animSequence structure. Next, the contents of the experimental parameter structure are copied back to the individual parameter variables, and the structure is disposed of. What follows is a parallel of the FilmFrames function of Listing 4, except that the animation frames are drawn directly by Picture instructions by calling the DrawPICTs function. Finally, the resource file is closed.

The DrawPICTs function transfers the animation frame images from a resource file into an array of GWorlds. For each animation frame in turn, the pixel map of the GWorld is drawn into simply by calling the DrawPicture routine, which is a ToolBox routine used to convert picture instruction sequences into pixel map images. The DrawPicture call is encircled by the appropriate memory locking and port-setting procedures required when accessing GWorld pixel maps.

Listing 6 concludes with two functions that free the memory allocated for the animSequence structure. DisposSequence frees all of the memory used by animSequence, whereas DisposFrames frees the memory used only by the animation frames themselves (the frames field of the animSequence). DisposSequence frees the memory of the PicsInfoRec with a call to DisposHandle. Next, the individual frames of the sequence are de-allocated via DisposFrames' use of the KillPicture routine, which destroys Picture structures specifically. The animSequence structure itself is then destroyed via DisposHandle.

\section{Conclusions}

Real-time color animation may be accomplished by methods other than frame animation. Baro and Hughes (1991) describe a "color-cycling" animation technique using the Palette Manager alone on a single image, which may be used to present many types of motion stimuli in real time. However, there are many classes of motion stimuli that cannot be accomplished by palette modifications alone. It is these stimuli that this paper addresses.

The use of frame animation outlined here for displaying real-time color animation has many features that are of use to vision scientists. Color-frame animation permits the presentation of moving gray-scale images at lower contrast levels than those using black-and-white graphics. The motion paths of the stimuli, as well as the stimuli shapes, are less constrained than those of palette-animated stimuli. For example, one may use frame animation to create an apparent motion stimulus that changes in shape or color. Frame animation is also well suited to the creation of random-dot motion stimuli. Although palette animation may be used to create such stimuli, their construction using a single image of several changing colors would be less straightforward than their construction using frame animation techniques. Stimuli such as drifting sinusoidal gratings, counterphasing checkerboards, or flickering fields are better suited to the use of palette animation. These two animation approaches are not mutually exclusive-both techniques may be combined to produce very complex moving stimuli.

The use of the GWorld structure affords more advantages than does the direct use of BitMaps or PixMaps. All three graphics structures help to bypass the drawing speed bottlenecks that prevent real-time drawing to the screen for anything other than simple black-and-white figures. However, only the GWorld simplifies the programmer's task of using color off-screen graphics by removing the need to manually keep pixel map, graphics device, and color table data synchronized. Only the GWorld helps to optimize the speed of the time-critical CopyBits routine. And only the GWorld allows the use of the simpler and more precise Palette Manager routines to keep the display color environment correct. Finally, Apple Computer encourages the use of GWorlds to ensure future program compatibility and because the GWorld routines are optimized for the new 8-24GC graphics accelerator board. However, the use of GWorlds requires that the Macintosh have 32-bit QuickDraw installed, either in ROM or in a software patch. Without it, the color-frame animation code will not run. It is the programmer's responsi- 
bility at the onset of the program to check the system environment via the Gestalt Manager for the presence of 32-bit QuickDraw.

By precomputing the graphics images, either programmatically or with a commercial paint package, the need to perform time-consuming drawing operations during the presentation of the animation is removed. At playback, only a simple, fast CopyBits operation is required, allowing larger, more complicated images to be used. By using a single transfer routine call to perform the animation, the Vertical Retrace Manager may be used for accurate timing of the animation presentation, ensuring precise visual motion velocities. The precomputed images are stored as Pictures (drawing instructions) rather than as pixel maps, reducing the storage needs as well. By making the animation files smaller, larger sets of stimuli may be stored and the time required to read each file from disk during a psychophysical experiment is shortened.

The use of resources provides both a simpler method of storing our graphics images and a means of saving the color environment so that it may be easily restored on playback. Saving and restoring the color environment ensures the maintenance of proper stimulus contrast or color and background luminance when displaying a previously designed stimulus. By also saving the experimental parameters as resources, the experimental parameters to be used with a given file may be examined later or modified to produce new stimuli.

Frame animation does, however, limit the complexity of the stimulus. The allowable physical size and color depth of the animation frames constrain the overall size of the stimulus and the number of gray levels that can be displayed at once. These limitations arise from the upper envelope of how much graphics memory may be transferred by the CopyBits routine during one vertical retrace interval. The larger the image and the higher its color depth, the longer it takes to transfer the image from offscreen memory to the screen. If the transfer time is not kept brief, the transfer may take more than one retrace period, slowing down the animation and introducing inaccuracies in visual motion velocity. When testing the animation code presented here on a Macintosh IIfx computer, it was found that when using 4-bit color (16-color) displays, real-time animation was possible only on images limited to $350 \times 350$ pixels in size. When 8-bit color (256color) displays were used, the largest possible image size for real-time animation was reduced to $230 \times 230$ pixels. The amount of available memory also places an upper limit on the image size, image depth, and the number of frames that may be displayed in an animation sequence.

Although the current code works correctly on the Macintosh IIfx, IIci, and IIsi, there is no guarantee that a color Macintosh with a slower CPU, such as the Macintosh lc, will be able to transfer large multicolored images quickly enough to sustain real-time animation. On the Macintosh lc, smaller images of fewer colors may be required, or the programmer may have to restrict animation to palette animation techniques alone. The installation of a QuickDraw accelerator board will not significantly in- crease the speed of the animation display because a single CopyBits call is executed within the vertical retrace interrupt task. However, the Apple 8*24GC graphics accelerator board permits the caching of GWorlds in the onboard video RAM, speeding up graphics transfers to the screen. This results in the ability to animate larger frame areas.

Despite the present restrictions on the use of color-frame animation, the techniques described here allow the creation of complex frame-animated motion stimuli in realtime on the Macintosh computer. ${ }^{3}$ The code listings provide a base with which a complete stimulus construction and presentation system may be constructed by adding drawing and user interface routines. Some of the techniques outlined here, such as the use of resources, may be used in other types of programs as well. The present restrictions on image size and color depth may be reduced or eliminated with the continued introduction of graphics accelerators, for example, the introduction of a "blitter" graphics coprocessor to relieve the CPU of the task of transferring graphics images. Yet even with present technology, color frame animation may be used alone or in combination with palette animation for a variety of visual psychophysics experiments.

\section{REFERENCES}

Anstis, S., \& Paradiso, M. (1989). Programs for visual psychophysics on the Amiga: A tutorial. Behavioral Research Methods, Instruments, \& Computers, 21, 548-563.

APPLE CoMPUTER (1985). Inside Macintosh: Volumes I-III. Menlo Park, CA: Addison-Wesley.

APple Computer (1991). Inside Macintosh: Volume VI. Menlo Park, CA: Addison-Wesley.

APPle CompUter (1984-1991). Macintosh technical notes. Cupertino, CA: Author.

Baro, J. A., \& Hughes, H. C. (1991). The display and animation of full-color images in real time on the Macintosh computer. Behavioral Research Methods, Instruments, \& Computers, 23, 537-545.

Ortiz, G. (1990). Braving offscreen worlds. Develop, 1, 28-40.

Rensick, R. A. (1990). Toolbox-based routines for Macintosh timing and display. Behavioral Research Methods, Instruments, \& Computers, $22,105-117$.

Sheets, S. (1988). Color screen dump FKEY. MacTutor, 4, 52-60. SHEETs, S. (1991). Animating PICS. MacTutor, 7, 64-69.

van Brink, D. (1990). All about the Palette Manager. Develop, 1, 22-27.

Wenderoth, P. (1990). Software-based visual psychophysics using the Commodore Amiga with Deluxe Paint III. Behavioral Research Methods, Instruments, \& Computers, 22, 383-388.

\section{NOTES}

1. The source code listings presented here were written in Think $\mathbf{C}$ version 5.0 (Symantec Corporation, Cupertino, $\mathrm{CA}$ ) and are compatible with the Macintosh System 7.0 operating system.

2. The duration of an animation frame was confirmed using millisecond-resolution timing routines (Rensick, 1990). An animation sequence was generated and displayed, with the timer started at the onset of the animation display and stopped at the end of the sequence. The total elapsed time was then divided by the number of frames to get the single frame time. A single frame was found to take $15 \mathrm{msec}$, the duration of a vertical retrace on the AppleColor RGB monitor.

3. The full source code of an animation demonstration program may be obtained from SBS. In addition, a demonstration version of a randomdot cinematogram psychophysics program based on this animation code may be requested. 


\section{LISTING 1}

FrameAnim.c

The main program file declares the major variables used in the animation program, depicted here as global variables. The InitParameters function initializes the animation and experimental parameters, and allocates memory for the animSequence structure that houses the Picture-format animation sequence. The CleanUp function frees up all memory allocated on the heap during the course of the program and restores the monitor color depth setting to its initial setting.

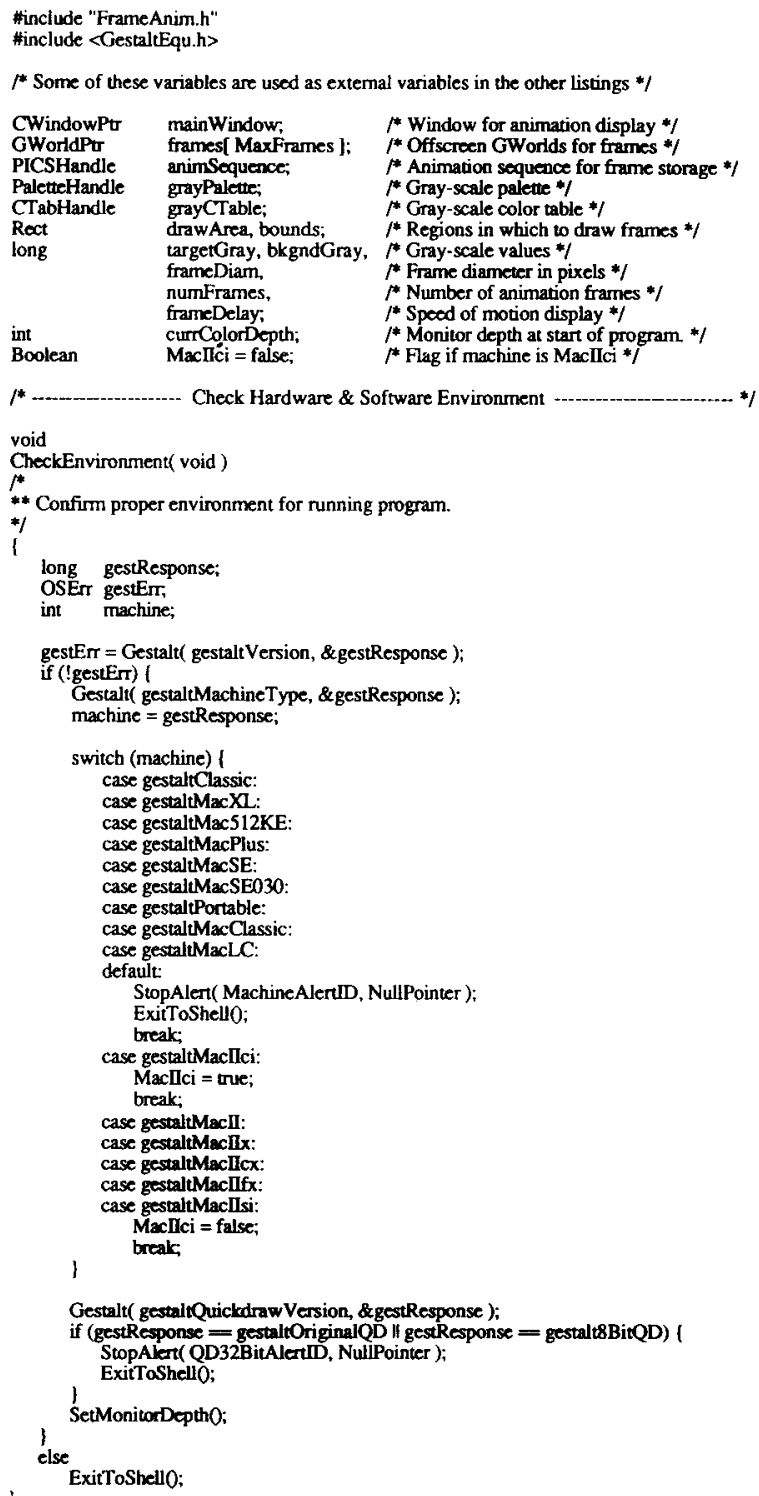

* Total number of frames filmed * * Wait 3 VBlanks between each frame display. */ * Frame diameter in pixels */

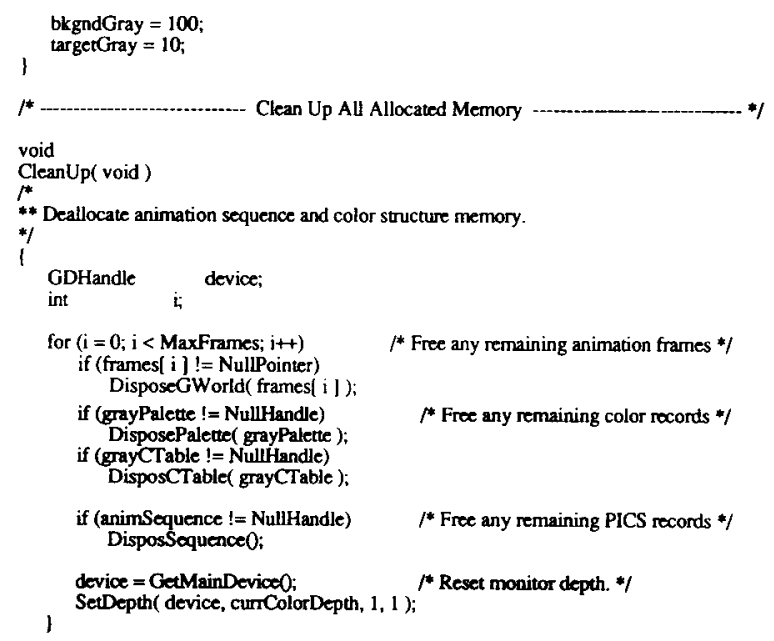

\section{LISTING 2}

FrameAnim.h

The program header file contains constant and type definitions. The constant definitions include resource ID numbers of the resources saved to disk in Listing 6 and of the generic grayscale ramp palette, color palette-specific definitions, and the vertical refresh rate. The type definitions specify the structures used to hold the animation frames (in Picture format) and relevant experimental parameters to be saved to disk. Function prototypes, required by Think C 5.0, are omitted in this listing.

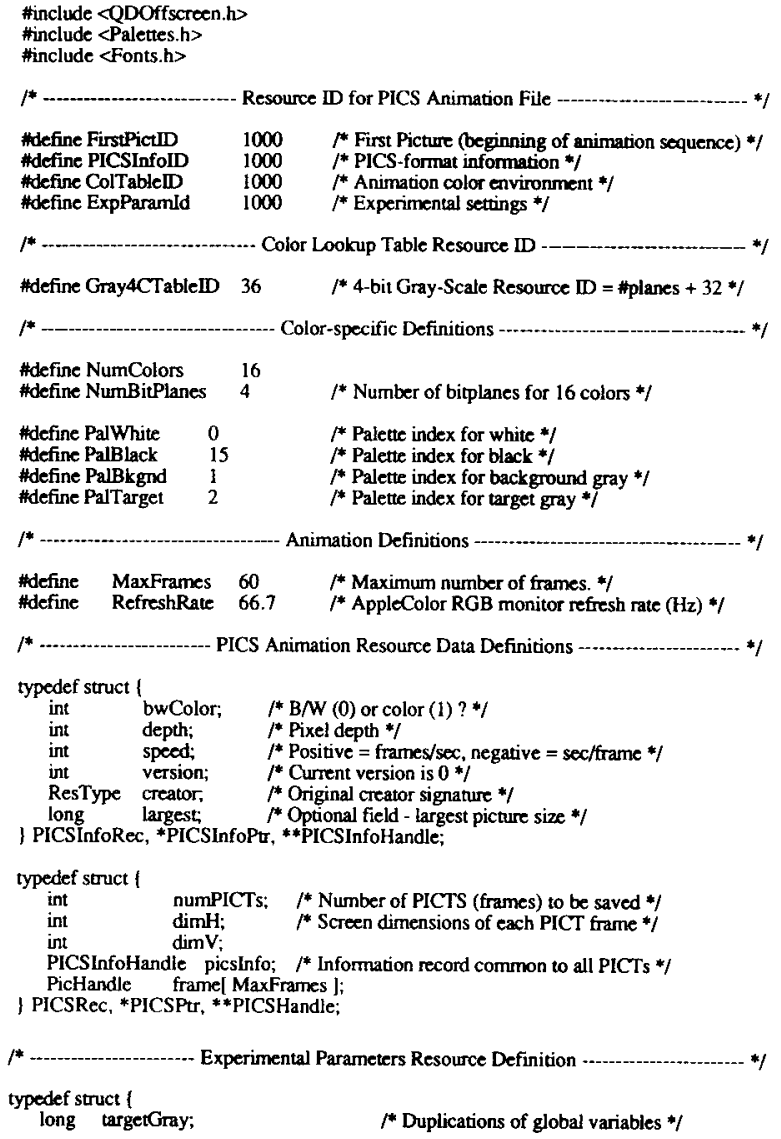




\author{
long bkgndGray \\ long frames; \\ long frameDelay: \\ | ExpParamRec, *ExpParamPtr, "*ExpParamHandle
}

\section{LISTING 3 Setup.c}

The SetMonitorDepth function sets the monitor color depth to four bitplanes. The SetupPalette function creates a gray-scale palette and color table. The display window for the frame animation sequence is assigned this palette and the palette is activated for use. UpdateWindowColors sets new gray values for the palette entries corresponding to the target and background colors and activates the modified palette. UpdateGWorldColors mirrors the palette changes made to the display window in the animation frames stored in the frames GWorld array.

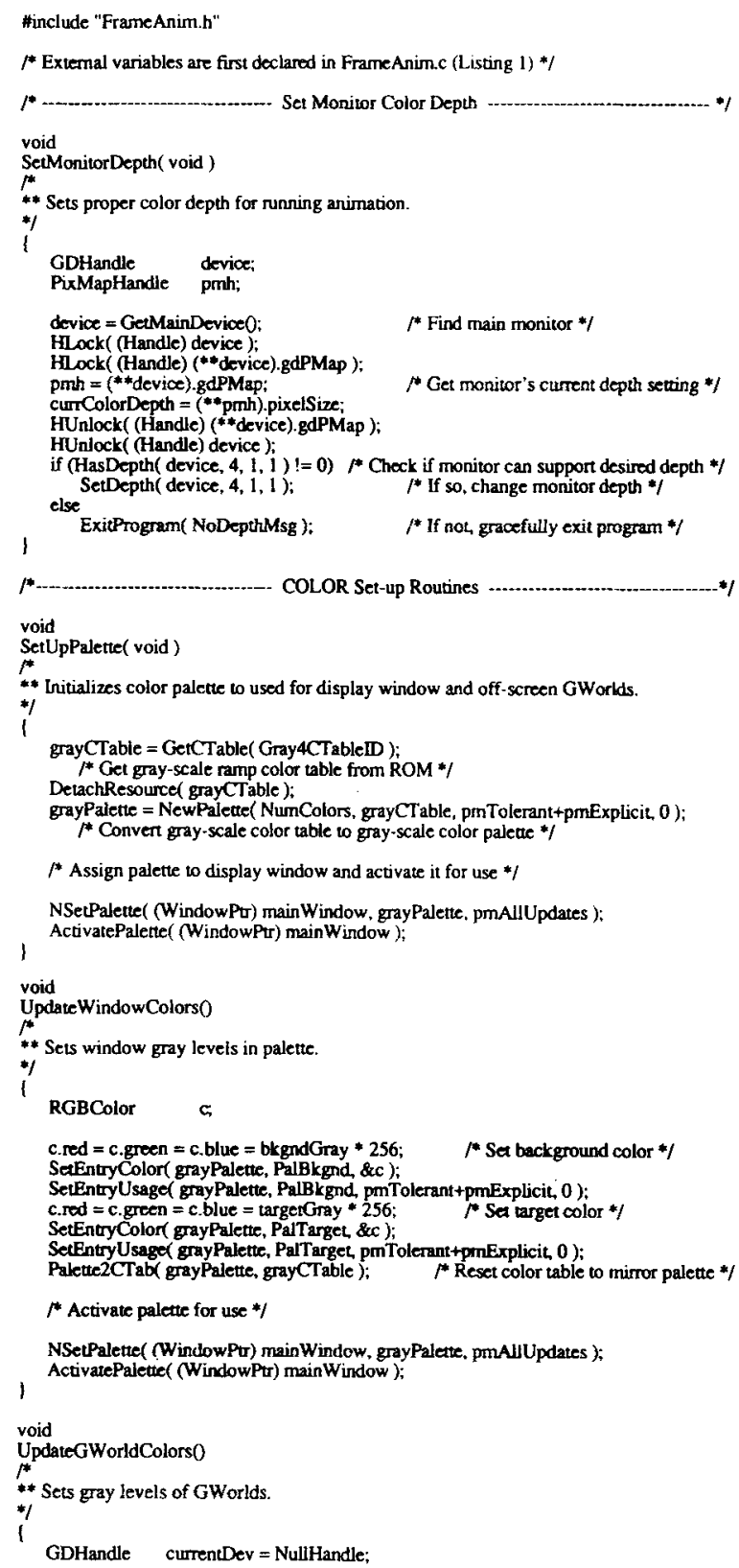

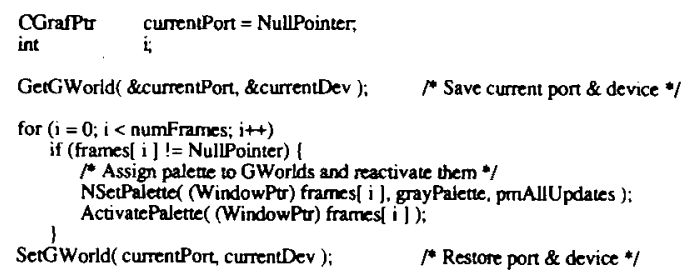

\section{LISTING 4 \\ Animation.c}

This listing contains the core routines of the animation program. The ReadyFilm function frees preexisting animation frame GWorlds and allocates memory for new GWorlds. FilmFrames oversees the frame creation process, calling DrawFrames to perform the actual creation of the images in each animation frame. The drawing operations are performed twice-once for storage in pixel map form in the GWorlds to be displayed on the screen and again for storage in Picture format to be saved as resources to disk. PlayFrames handles the display of the animation sequence via the video interrupt task procedure. The ResetForeAndBackColors function simply sets the foreground and background drawing pen colors required for proper operation of the CopyBits routine used in Listing 5.

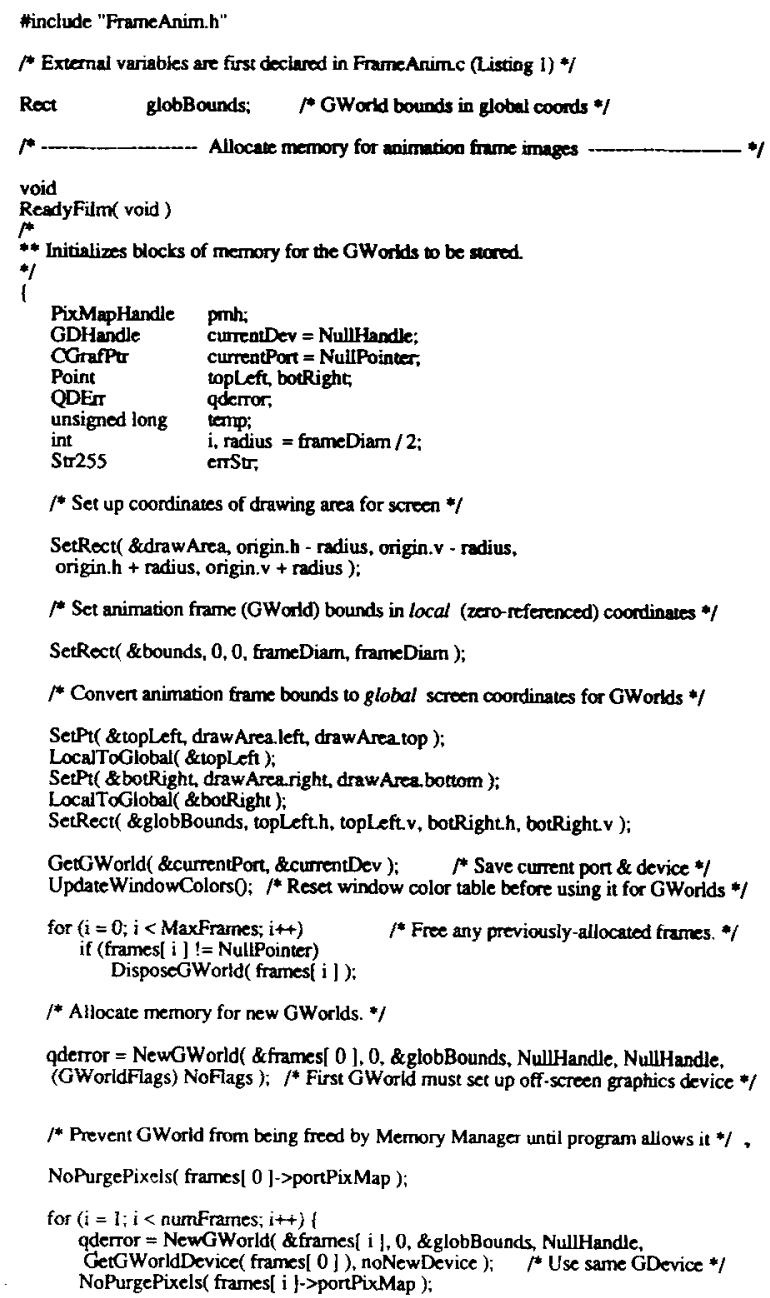




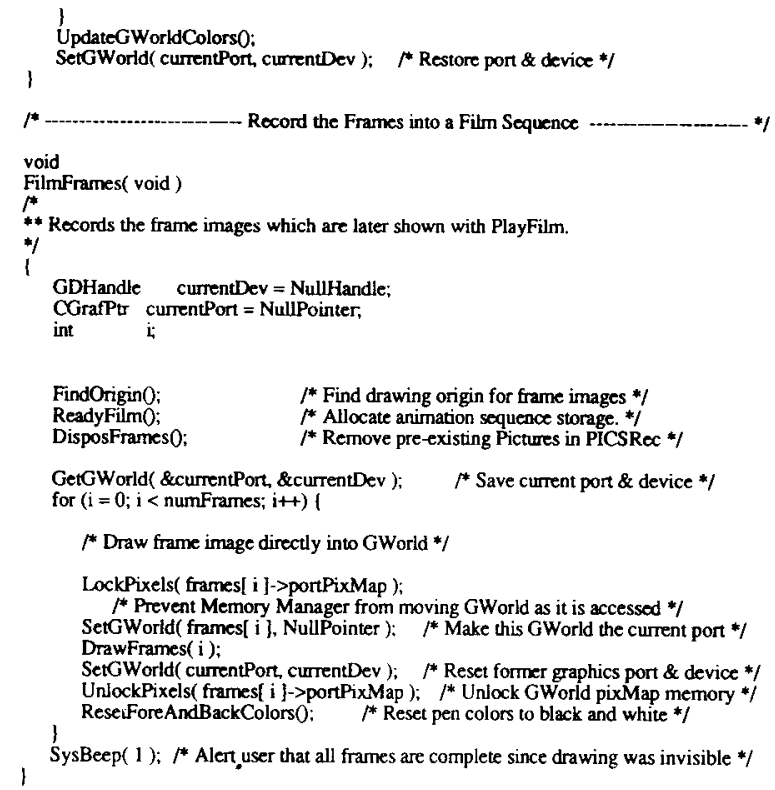

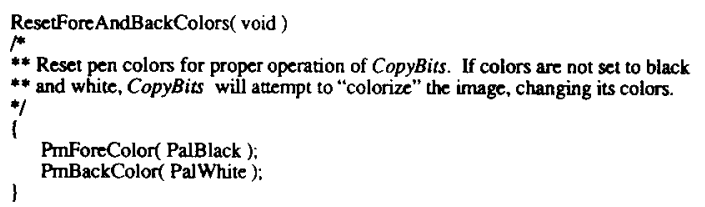

\section{LISTING 5 \\ VBlank.c}

The InstallVBlankAnim function initializes the video interrupt task (VBLTask) and slot interrupt task (SlotIntQElement) structures, and inserts the VideoProc and SlotProc tasks into the vertical interrupt and slot interrupt queues, respectively. The VideoProc vertical retrace interrupt task is called at a rate specified by the frameDelay variable. The SlotProc procedure simply calls the VideoProc function. Within VideoProc, the contents of one frame's offscreen graphics structure is copied to the screen. When the animation has completed, the RemoveVBlankAnim function removes the VideoProc and SlotProc tasks from their respective interrupt queues.

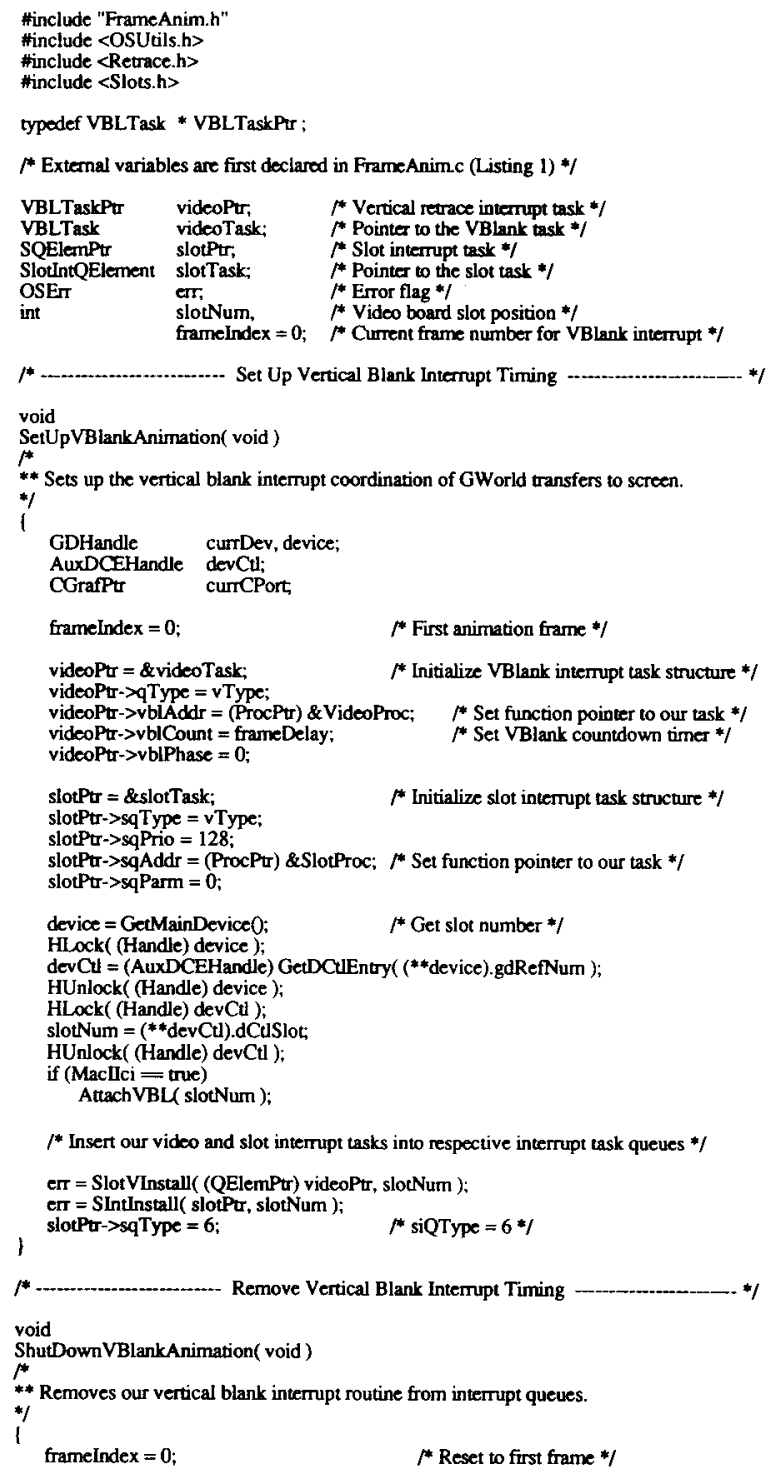




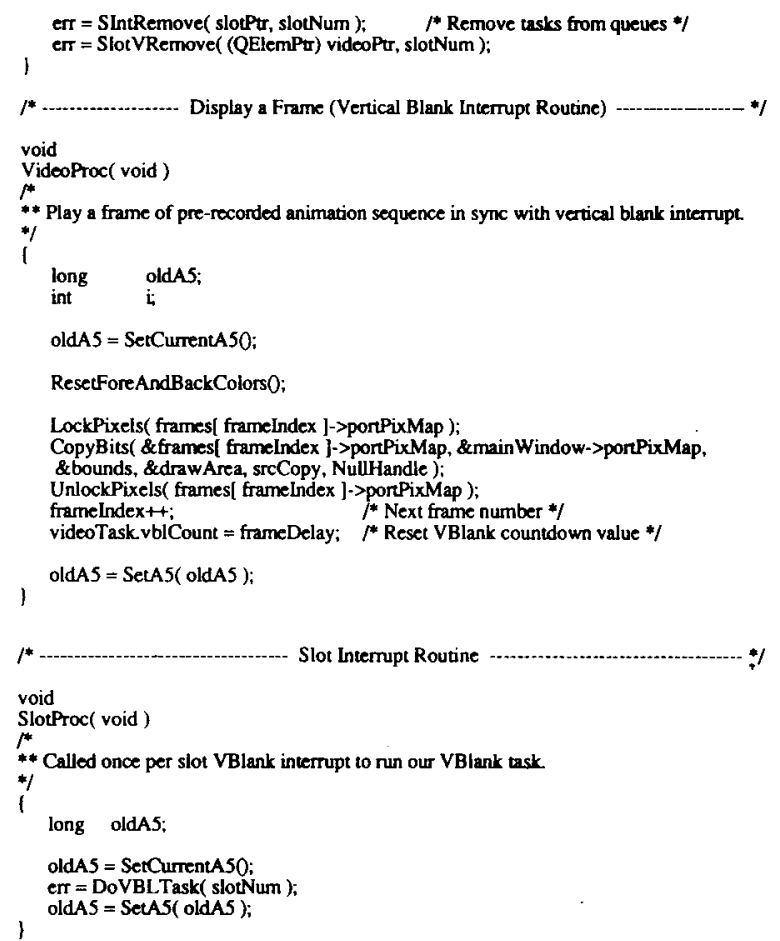

\section{LISTING 6 Files.c}

The Writelmage function transfers the experimental parameters, the animation frame images, and the color table to a newlycreated resource file. ReadImage performs the complementary action of reading the contents of these resources back from the disk file. The Pictures read from the file are drawn into individual off-screen GWorlds for display via the DrawPICTs function. DisposSequence and DisposFrames free the memory allocated for either the entire animation sequence or the animation frames alone, respectively.

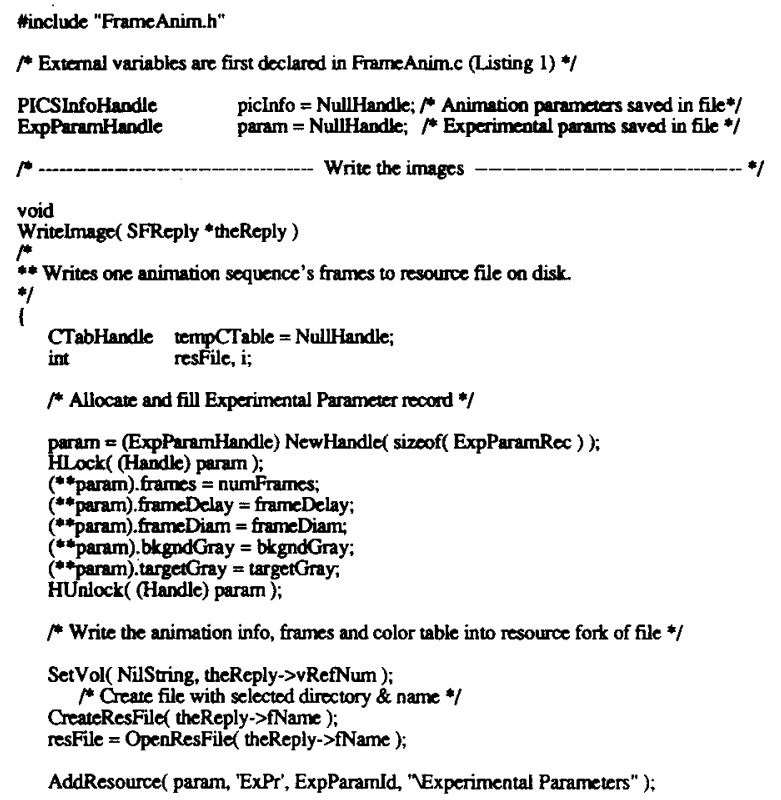

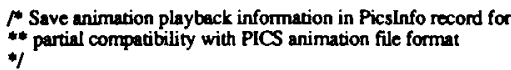


HLock ( (Handle) animSequence );

(**animSequence).framel i ] = GetPicture (FirstPictlD + i);

DetachResource ( ${ }^{* *}$ animSequence).frame $[\mathrm{i}]$ );

)

HUnlock( (Handie) animSequence);

1 * Copy animation frame Pictures into off-screen GWorlds for display */

FindOrigino:

ReadyFilmO:

$\begin{array}{ll}\text { DrawPICTs0; } & { }^{*} \text { Transfer the frame image Pictures into the GWorlds. */ } \\ \text { ResetBackground0; } & * \text { Reser screen background to prepare for animation display */ }\end{array}$

$f^{*}$ Find screen placements for each frame image. */

* Allocate off-screen GWorld storage \& update palettes. */

CloseResFile( resFile ); $\quad *$ Close resource file *j

I

picInfo = NullHandle;

$/ *$

void

DrawPICTs( void)

** Draw the contents of the resource file into the animation frame image GWorlds.

*1

GDHandle currentDev = NullHandle:

$\begin{array}{ll}\text { GDHandle } & \text { currentDev }=\text { NullHandle; } \\ \text { CGrafPtr } & \text { currentPort }=\text { NullPointer }\end{array}$

I* Save current port \& device *I

for $(i=0 ; i<$ numFrames; $i++)$ (

if ( (**anim Sequence) frame $[i] !=$ NullHandle)

HLock ( (Handle) animSequence); $/ *$ Lock all memory to be accessed */

HLock( (Handle) (**animSequence). frame[ i ]);

HLock (Handle) frames[ i ]);

LockPixels( frames[ i ]->portPixMap)

SetGWorld( frames[ i ], NullPointer ); $/ *$ Make GWorld the current port *I

1 * Transfer the contents of each Picture to an off-screen GWorld pixMap */

DrawPicture( (**animSequence).frame[ i ], \&bounds );

SetGWorld( currentPort, currentDev); $\quad$ * Reset former port \& device */

UnlockPixels ( frames[ $\mathrm{i}$ ]->portPixMap ); $/^{*}$ Unlock all memory */

HUnlock( (Handle) frames[ i ] );
1

HUnlock( (Handle) (**animSequence).frame[ i ]);

Hunlock( (Handle) animSequence )

void
DisposSequence (void)

** Dispose of the entire animation sequence structure, including all Pictures.

i

DisposFrames 0

f ( (**animSequence).picsinfo != NullHandle $)$

DisposHandle ( (Handle) (**arimSequence).picsInfo)

(animSequence != NullHandie)

l*

DisposHandle( (Handle) animSequence );

void

DisposFrames ( void )

** Dispose of the animation record frame Pictures only.

*1

int $\quad \mathrm{i}$

for $(i=0$; $i<$ MaxFrames; $i++)$

if ( $(* *$ animSequence) frame[ $i] !=$ NullHandle)

HLock (Handle) animSequence);

KillPicture ( $(* *$ animSequence).frame[ i ] );

(**animSequence).frame[ $i]$ = NullHandle-

HUnlock ( (Handle) animSequence )
Huans

(Manuscript received May 13, 1991;

revision accepted for publication January 9, 1992.) 